\title{
Characterization of carriers in GaSb/InAs superlattice grown on conductive GaSb substrate
}

\author{
T. V. Chandrasekhar Rao, ${ }^{a)}$ J. Antoszewski, and L. Faraone \\ School of Electrical, Electronic and Computer Engineering, The University of Western Australia, Crawley, \\ Western Australia 6009, Australia \\ J. B. Rodriguez, E. Plis, and S. Krishna \\ Center for High Technology Materials, Department of Electrical and Computer Engineering, \\ University of New Mexico, Albuquerque, New Mexico 87106, USA
}

(Received 16 November 2007; accepted 13 December 2007; published online 11 January 2008)

\begin{abstract}
We report on mobility spectrum analysis of electrical transport in a $\mathrm{GaSb} / \mathrm{InAs}$ superlattice (SL) grown on $\mathrm{GaSb}$ substrate. Despite domineering contribution to conduction from the substrate, it was possible to discern and characterize carriers from SL. A single electron specie with an ambient temperature mobility of $\sim 10^{4} \mathrm{~cm}^{2} / \mathrm{V}$ s was found to emanate from SL. We show that this carrier has an activation energy of $0.27 \mathrm{eV}$ and is associated with the SL band gap. (C) 2008 American Institute of Physics. [DOI: 10.1063/1.2831666]
\end{abstract}

Photodiodes based on InAs/ $\mathrm{Ga}(\mathrm{InSb})$ short period superlattices (SLs) epitaxially grown on GaSb substrates have emerged in the past few years as a promising technology. ${ }^{1}$ Several research groups have demonstrated high performance photodiodes both in the midinfrared atmospheric window $(3-5 \mu \mathrm{m})$ as well as the long-wave range $(8-12 \mu \mathrm{m}){ }^{2}$ including a dual-color midinfrared (mid-IR) focal plane array $^{3}$ and operation at room temperature. ${ }^{4}$ In order to further improve ambient temperature performance of detector material in terms of issues such as collection efficiency, one needs to have a better understanding of underlying carrier transport in this material. However, electrical transport measurements on these structures are difficult, especially at high temperatures, as the SLs are grown on highly conductive GaSb substrates. Commercially available $\mathrm{GaSb}$ substrates have, at best, a residual doping level of low $10^{17} / \mathrm{cm}^{3}$ and substrates being significantly thicker than SLs, carriers from the former will have an overbearing contribution to electrical transport. It is, however, possible to make some indicative measurements on thick SLs grown on semi-insulating GaAs substrates, but a strong lattice mismatch, leading to a large defect density, puts an onus on applicability of those results to GaSb based SLs. Some groups have reported Hall measurements by introducing a lattice matched quaternary (AlGaAsSb) buffer layer between the substrate and the superlattice, ${ }^{5}$ but this technique is only suitable at low temperatures where quaternary layer conductivity is sufficiently low. Temperature dependant capacitance-voltage measurements on " $p-i-n$ " SL-based diodes, where " $i$ " refers to unintentionally doped region, have been reported ${ }^{6}$ and provide interesting values of background carrier concentration between 20 and $200 \mathrm{~K}$. However, capacitance-voltage measurements do not provide complimentary information such as mobility and polarity of carriers.

On the other hand, the method of "quantitative mobility spectrum analysis (QMSA)" is a powerful tool for analyzing electrical transport in materials with multiple carriers of

\footnotetext{
${ }^{a)}$ On leave from Technical Physics and Prototype Engineering Division, Bhabha Atomic Research Centre, Bombay 400085, India. Electronic mail: chandra1@barc.gov.in.
}

varying contribution to sample conduction. ${ }^{7}$ Since high mobility carriers get turned off at high magnetic fields, conductivity tensor components of the sample obtained under multiple magnetic fields can be resolved into contributions from various carriers. QMSA algorithm converts experimentally measured resistivity and Hall coefficient data into "carrier mobility spectra," showing conductivity as function of carrier mobility, ${ }^{8}$ with peaks corresponding to every carrier specie present. A cumulative analysis of all mobility channels under a peak gives us density and mobility of the carrier concerned,

$$
n_{i}=\frac{d}{q} \sum_{j=1}^{N} \frac{\sigma_{\mathrm{j}}}{\mu_{j}} \quad \text { and } \quad \mu_{i}=\frac{1}{N}\left(\sum_{j=1}^{N} \mu_{j}\right),
$$

where $n_{i}$ and $\mu_{i}$ are the sheet density and mobility of $i^{\text {th }}$ carrier, $N$ is the total number of mobility channels under $i^{\text {th }}$ spectral peak, $d$ is sample thickness, and $q$ is the electron charge. In this letter, we report the use and results of QMSA to study in-plane conduction in a $\mathrm{GaSb} / \mathrm{InAs} / \mathrm{GaSb} \mathrm{SL}$ sample (with a cutoff wavelength of $\sim 5.4 \mu \mathrm{m}$ at $300 \mathrm{~K}$ ). Through a comparative analysis of measurements on SL sample as well as on bare substrate, we extract the properties of the SL layer in the temperature range 100 to $300 \mathrm{~K}$.

InAs/ GaSb material was grown epitaxially on an undoped, $490 \mu \mathrm{m}$ thick, (001) GaSb substrate using a solid source VG80 molecular beam epitaxy reactor, with $\mathrm{As}_{2}$ (valved) and $\mathrm{Sb}_{2}$ cracker sources. Growth rates were set, respectively, to 0.33 and $0.5 \mathrm{ML}$ for InAs and $\mathrm{GaSb}$, while the corresponding $V$-III flux ratios were 2.4 and 5.0. As and $\mathrm{Sb}$ soak times of 2 and $12 \mathrm{~s}$ were used for InAs and $\mathrm{GaSb}$ layers, respectively, in order to sharpen the interfaces. A 500 period SL made up of $9 \mathrm{ML}$ of GaSb and $9 \mathrm{ML}$ of InAs was deposited on $\mathrm{GaSb}$ surface at $\sim 400{ }^{\circ} \mathrm{C}$. The nominal total thickness of the sample was thus $2.7 \mu \mathrm{m}$. We used $(1 \times 3)$ to $(2 \times 5)$ reflection high-energy electron diffraction reconstruction transition temperature $\left(\sim 450{ }^{\circ} \mathrm{C}\right)$ observed on $\mathrm{GaSb}$ surface as a reference for all growth temperatures. Details about growth conditions have been published elsewhere. ${ }^{9}$ Figure 1 shows the high-resolution x-ray diffraction scan obtained for this sample. Intense and numerous satellite peaks 


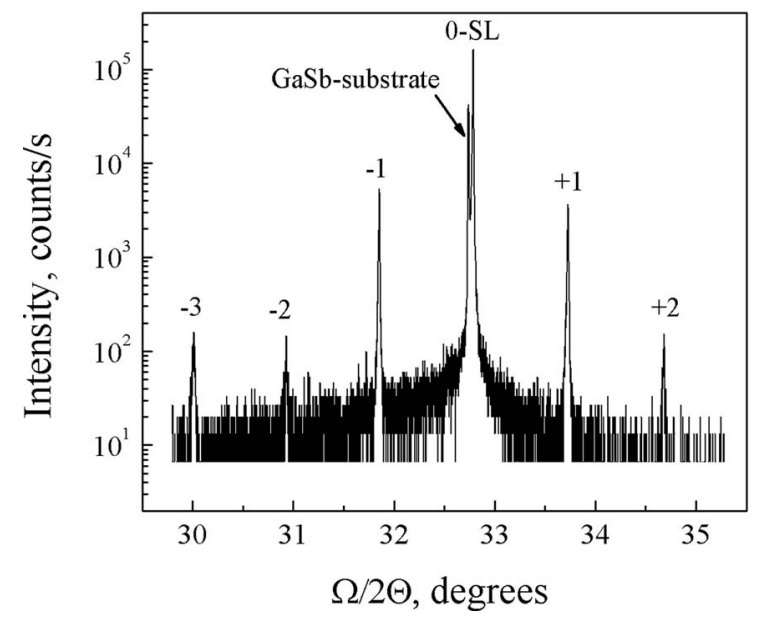

FIG. 1. X-ray diffraction pattern of $9 \times 9 \mathrm{MLs}^{2} \mathrm{InAs} / \mathrm{GaSb}$ superlattice grown on GaSb. From peak spacing, SL period is estimated to be $5.4 \mathrm{~nm}$.

are visible, attesting its good crystalline quality and layer reproducibility. From the spacing of satellite peaks we note that each period is $5.4 \mathrm{~nm}$ thick, in good agreement with the nominal value. We have also estimated the lattice mismatch of the epitaxial layer to be $0.13 \%$. For similar structures, using atomic force microcopy, we have noted rms value of surface roughness to vary between 0.2 and $0.4 \mathrm{~nm}$.

Hall voltage and resistivity measurements were undertaken on a sample of area $5 \times 5.5 \mathrm{~mm}^{2}$ with electrical contacts at the corners of SL layer in Van der Pauw configuration. Ohmic contact pads consisting of $\mathrm{Ti}, \mathrm{Pt}$, and $\mathrm{Au}$ were deposited by thermal evaporation. To evaluate substrate contribution to sample conduction, separate measurements were made under identical conditions on a $440 \mu \mathrm{m}$ thick piece of $\mathrm{GaSb}$ of similar area, after mechanically removing the SL. A superconducting magnet (M/S. Oxford Instruments) provided variable magnetic field $(0$ to $\pm 10 \mathrm{~T})$, while sample temperature was varied between 100 and $300 \mathrm{~K}$ using a liquid helium cryostat. The conductivity and Hall voltage measurements were made using a constant current of $10 \mathrm{~mA}$ and voltage drops were measured across appropriate pairs of contacts according to standard switching procedures. The data was analyzed with Lakeshore I-QMSA package, based on an algorithm outlined by Vurgaftman et al., ${ }^{7}$ leading to mobility spectra showing density and mobility of all contributing carrier species (Fig. 2).

Hall coefficient was positive for the entire temperature window investigated, showing hole dominance of conductivity originating from GaSb substrate. ${ }^{10}$ For most temperatures, mobility spectra of SL sample showed five carrierstwo holes and three electrons-labeled $h_{1}, h_{2}, e_{1}, e_{2}$, and $e_{3}$, as shown in Fig. 2(a), obtained at $100 \mathrm{~K}$. Measurements on bare substrate at the same temperature show the presence of four of those carriers [Fig. 2(b)], $e_{1}$ being the specie absent. Hence, we infer that $e_{1}$ emanates from the SL layer while other carriers arise from GaSb substrate. In order to characterize $e_{1}$, we have estimated its sheet density in the region 100-300 K using Eq. (1) and plotted the same as a function of inverse temperature, as shown in Fig. 3. From the slope of the plot at higher temperatures $(T>250 \mathrm{~K})$, we have estimated the energy barrier associated with $e_{1}$ to be about $0.27 \mathrm{eV}$, in good agreement with theoretically computed band gap of a $8 \times 8$ superlattice $^{11}$ and photoluminescence data. $^{12}$ Besides, excitation of $e_{1}$ electrons across the SL gap

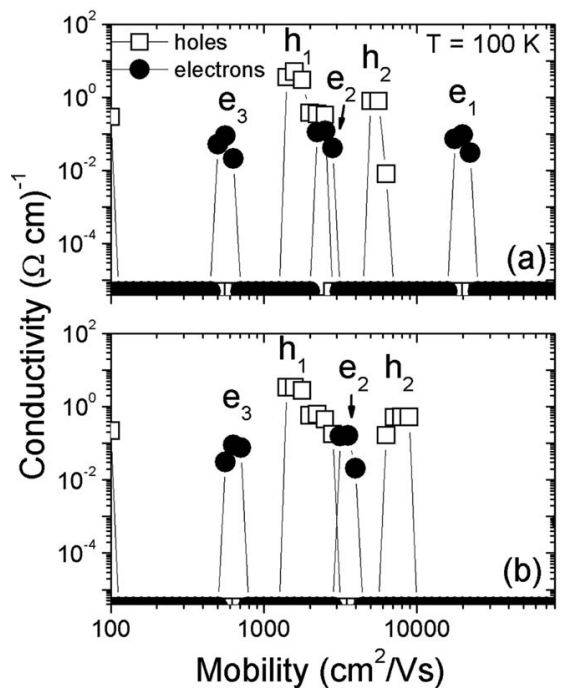

FIG. 2. (a) Mobility spectrum of $\mathrm{GaSb} / \mathrm{InAs} / \mathrm{GaSb}$ superlattice sample obtained at $100 \mathrm{~K}$ using QMSA procedures. A total of five carrier species can be seen: three electrons $e_{1}, e_{2}$, and $e_{3}$ (solid circles) and two holes $h_{1}$ and $h_{2}$ (open squares). (b) Mobility spectrum of bare GaSb substrate, also at $100 \mathrm{~K}$. Note the absence of carrier $e_{1}$.

should create holes in its valance band, leading to hole conduction within SL. We note an increase in the density of low mobility holes $h_{1}$ for $T>250 \mathrm{~K}$, over and above substrate contribution (see inset of Fig. 3), which is a definite signature of such an enhancement in hole conductivity. In addition, mobility of electron $e_{1}$ evaluated using Eq. (1) is shown as a function of temperature in Fig. 4. Its ambient temperature value $\left(\sim 10^{4} \mathrm{~cm}^{2} / \mathrm{V} \mathrm{s}\right)$ matches well with that reported in literature for a similar SL system. ${ }^{5}$ For $T>100 \mathrm{~K}$, mobility scales as $\mu \sim T^{-1.23}$ in agreement with theoretically expected exponent value of " 1.5 " due to mobility degradation caused by phonon scattering, ${ }^{13}$ and an experimental value of "1.4" reported for GaSb. ${ }^{14}$ The drop in mobility at low temperatures is a result of ionized impurity scattering, as noted earlier. ${ }^{15}$ Thus, results obtained by QMSA clearly show that the electron specie $e_{1}$ arises from the $\mathrm{GaSb} / \mathrm{InAs}$ superlattice and electrical conduction within SL is linked to thermal excitation of carriers across its bandgap of $0.27 \mathrm{eV}$.

Finally, we use temperature dependent QMSA to characterize carriers emanating from GaSb substrate as well. Hole $h_{1}$, with ambient temperature concentration $(n)$ of $7.1 \times 10^{15} / \mathrm{cm}^{2}$ and a mobility $(\mu)$ of $600 \mathrm{~cm}^{2} / \mathrm{V} \mathrm{s}$, has an

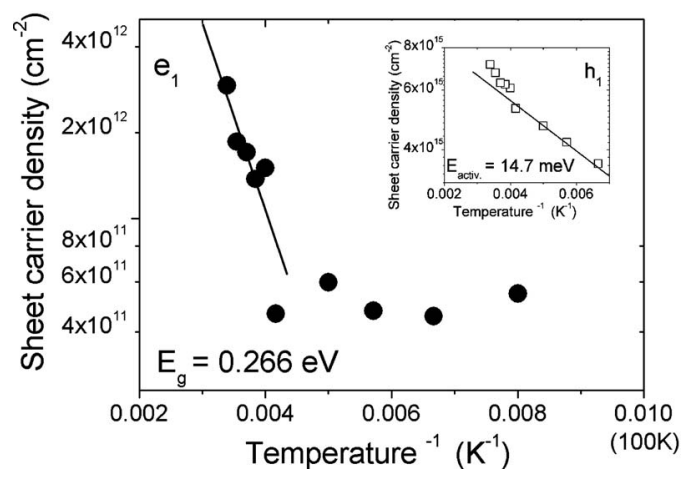

FIG. 3. Sheet carrier density of $e_{1}$ estimated using QMSA, shown as a function of inverse temperature. Band gap is estimated by a linear fit to the data in the range $260-300 \mathrm{~K}$. Inset shows an increase in hole concentration for $T>250 \mathrm{~K}$. 


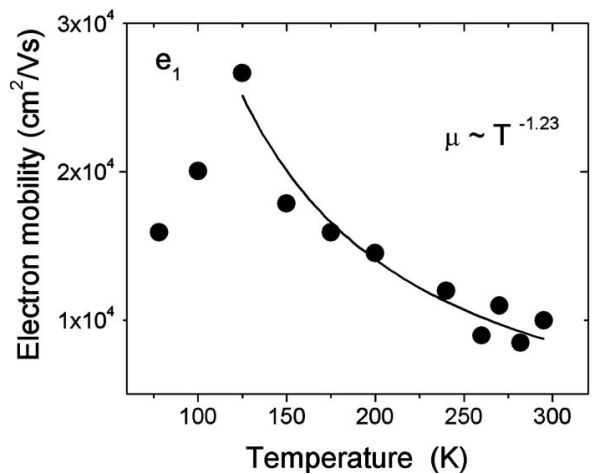

FIG. 4. Temperature dependence of the mobility of $e_{1}$, estimated using QMSA. The degradation at higher temperatures is a consequence of phonon scattering.

activation energy $\left(E_{a}\right)$ of $14.7 \mathrm{meV}$ (see inset of Fig. 3). It arises from a native defect in $\mathrm{GaSb}\left(V_{\mathrm{Ga}}-\mathrm{Ga}_{\mathrm{Sb}}\right.$ pair $)$ as reported earlier. ${ }^{13}$ The second hole specie $h_{2}$ has $E_{a}$ $=40 \mathrm{meV}$ and could be associated with $\mathrm{Zn}$ acceptors in the substrate. ${ }^{16}$ Its $n$ and $\mu$ values, respectively, are 2.1 $\times 10^{14} / \mathrm{cm}^{2}$ and $2500 \mathrm{~cm}^{2} / \mathrm{V} \mathrm{s}$. Of the two electron species noted, parameters for $e_{3}$ are $E_{a}=53 \mathrm{meV}, \quad n=1.7$ $\times 10^{14} / \mathrm{cm}^{2}$ and $\mu=960 \mathrm{~cm}^{2} / \mathrm{V} \mathrm{s}$ and is probably linked to Se donor atoms. ${ }^{16}$ Electron $e_{2}$ has $E_{a}=30 \mathrm{meV}, n=2.6$ $\times 10^{14} / \mathrm{cm}^{2}$ and $\mu=260 \mathrm{~cm}^{2} / \mathrm{V} \mathrm{s}$ at $200 \mathrm{~K}$.

In conclusion, we have studied the nature of in-plane electrical transport in GaSb/InAs SL using mobility spectrum approach stemming from variable magnetic field measurements. As SL was grown on a conductive and thick $\mathrm{GaSb}$ substrate, our results expectedly show that sample conduction is dominated by carriers arising from the substrate. However, we have identified an electron specie arising from the SL, associated with its band gap of $0.27 \mathrm{eV}$. Future mea- surements will involve transport in the SL along the growth direction.

Support is acknowledged from DARPA-HOT MWIR Program and AFRL Contract No. FA9453-07-C-0171. Two of the authors (T.V.C.R. and S.K.) would like to acknowledge support from the Gledden Foundation.

${ }^{1}$ A. Rogalski and P. Martyniuk, Infrared Phys. Technol. 48, 39 (2006).

${ }^{2}$ H. Mohseni, E. Michel, J. Sandoen, M. Razeghi, W. Mitchel, and G. Brown, Appl. Phys. Lett. 71, 1403 (1997).

${ }^{3}$ R. Rehm, J. Schmitz, J. Fleißner, M. Walther, J. Ziegler, W. Cabanski, and R. Breiter, Phys. Status Solidi C 3, 435 (2006).

${ }^{4}$ Y. Wei, A. Hood, H. Yau, A. Gin, M. Razeghi, M. Z. Tidrow, and V. Nathan, Appl. Phys. Lett. 86, 233106 (2005).

${ }^{5}$ L. Bürkle, F. Fuchs, J. Schmitz, and W. Pletschen, Appl. Phys. Lett. 77, 1659 (2000).

${ }^{6}$ A. Hood, D. Hoffman, Y. Wei, F. Fuchs, and M. Razeghi, Appl. Phys. Lett. 88, 052112 (2006).

${ }^{7}$ I. Vurgaftman, J. R. Meyer, C. A. Hoffman, D. Redfern, J. Antoszewski, L. Faraone, and J. R. Lindemuth, J. Appl. Phys. 84, 4966 (1998).

${ }^{8}$ J. Antoszewski and L. Faraone, Opto-Electron. Rev. 12, 347 (2004).

${ }^{9}$ E. Plis, S. Annamalai, K. T. Posani, S. Krishna, R. A. Rupani, and S. Ghosh, J. Appl. Phys. 100, 014510 (2006).

${ }^{10}$ H. J. Haugan, G. J. Brown, F. Szmulowicz, L. Grazulis, W. C. Mitchel, S. Elhamri, and W. D. Mitchell, J. Cryst. Growth 278, 198 (2005).

${ }^{11}$ F. Szmulowicz, H. Haugan, and G. J. Brown, Phys. Rev. B 69, 155321 (2004).

${ }^{12}$ A. P. Ongstad, R. Kaspi, C. E. Moeller, M. L. Tilton, D. M. Gianardi, J. R. Chavez, and G. C. Dente, J. Appl. Phys. 89, 2185 (2001).

${ }^{13}$ R. D. Wiersma, J. A. H. Stotz, O. J. Pitts, C. X. Wang, M. L. W. Thewalt, and S. P. Watkins, Phys. Rev. B 67, 165202 (2003).

${ }^{14}$ P. S. Dutta, V. Prasad, H. L. Bhat, and V. Kumar, J. Appl. Phys. 80, 2847 (1996).

${ }^{15}$ J. H. Roslund, K. Saito, K. Suzuki, H. Yamaguchi, and Y. Hirayama, Jpn. J. Appl. Phys., Part 1 39, 2448 (2000).

${ }^{16} \mathrm{http}: / / \mathrm{www}$.ioffe.rssi.ru/SVA/NSM/Semicond/GaSb/bandstr.html and references therein. The site provides activation energies of several shallow donors and acceptors of $\mathrm{GaSb}$ and links to original references. 
Applied Physics Letters is copyrighted by the American Institute of Physics (AIP). Redistribution of journal material is subject to the AIP online journal license and/or AIP copyright. For more information, see http://ojps.aip.org/aplo/aplcr.jsp 